\title{
From the cradle
}

Normal brain function depends on the appropriate formation of neuronal networks during development. Genetic susceptibility factors to psychiatric disorders have been postulated to act by introducing slight perturbations to this neurodevelopmental programme that may lead to the manifestation of symptoms later in life. Now, Niwa et al. provide evidence for this hypothesis by showing

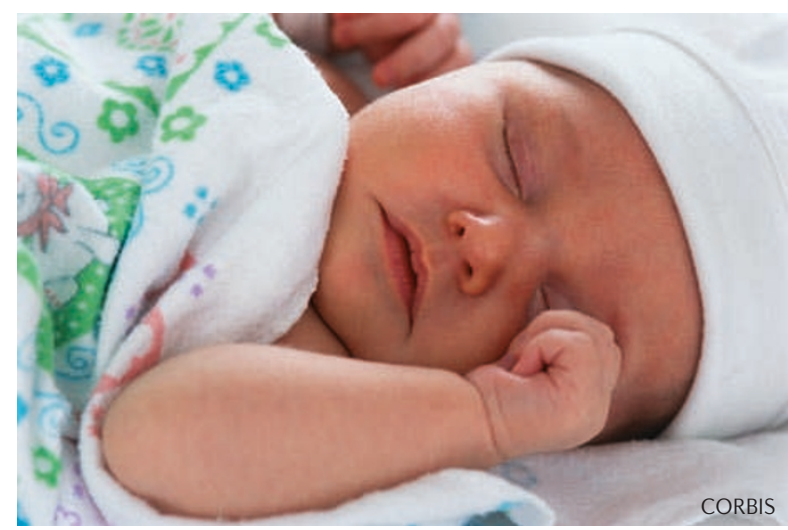

that transient in utero knock down of disrupted in schizophrenia 1 (Disc1) in mice causes abnormalities in mesocortical dopaminergic maturation and behavioural impairments after puberty.

The authors used in utero transfer of short interfering RNA to transiently suppress the expression of Disc1 during the last week of intra-uterine development. The timing of electroporation allowed them to specifically target precursors of pyramidal neurons in the prefrontal cortex (PFC), resulting in abnormalities in the dendritic morphology and electrophysiological properties of PFC neurons at postnatal day 14 . As the animals matured, the extracellular dopamine levels in the medial PFC became lower than in controls, as did immunoreactivity against tyrosine hydroxylase. In behavioural tests, the adult mice exhibited deficits in prepulse inhibition and in a novel-object recognition task, but this was normalized when dopamine levels were increased by acute administration of clozapine. Finally, the mice exhibited hypersensitivity to methamphetamines, further suggesting a specific deficit in dopaminergic signalling.

These results provide compelling evidence for a sequential link between transient deficits in gene expression during early development, postnatal dendritic abnormalities and altered behaviour later in life. This has important implications for our understanding of the genetic components of psychiatric disorders. Future studies are likely to provide further clues about this cascade, and the stages that could be targeted for early diagnosis and therapeutic intervention.

\section{Cristian Bodo}

ORIGINAL RESEARCH PAPER Niwa, M. et al. Knockdown of DISC 1 by in utero gene transfer disturbs postnatal dopaminergic maturation in the frontal cortex and leads to adult behavioral deficits. Neuron 65, 480-489 (2010) 